\title{
Drahtlose Beschleunigungsmessungen, Schwingungs- und Schockmessungen mit Hilfe von RFID-Transpondern
}

Reinhard Jurisch

microsensys $\mathrm{GmbH}$, In der Hochstedter Ecke 2, 99098 Erfurt

+49361598740, rjurisch@microsensys.de

RFID - Radio Frequency Identification - ist eine bekannte und inzwischen bewährte Auto-ID Technologie für kontaktlose Identifikation und Datenspeicherung am Objekt. Die Grundidee diese Technologie auch als drahtlose Schnittstelle für die Speisung und Datenübertragung von Sensoren zu nutzen ist nicht neu. Produktvisionen und diverse Realisierungen sind aus der Literatur bereits Anfang der neunziger Jahre bekannt [1], [2], [3].

Die Basis für komfortable Transponder, als Kernelement dieser RFID Systeme, bildet ein einziger Siliziumchip, der sich durch ein absolutes Low Power Mixed Signal Design auszeichnet. Ein Trend zur Systemintegration und zur Siliziumtechnologie ist seit Jahren auch im Sensorelementbereich zu verzeichnen. Es liegt damit nahe, Sensorik und RFID in einem Bauelement zu vereinen. Bekannt sind hierbei Lösungen, die dies für die physikalischen Größen Druck, Temperatur und Feuchte bereits umsetzen [4], [5], [6], [7]. Die Systemstruktur (siehe Abb. 1) solcher Anordnungen wiederholt sich vorteilhafterweise identisch, sodass bei einem bekannten und standardmäßigen AIR-Interface identische Reader-Technik und HOST-Systeme benutzt werden können.

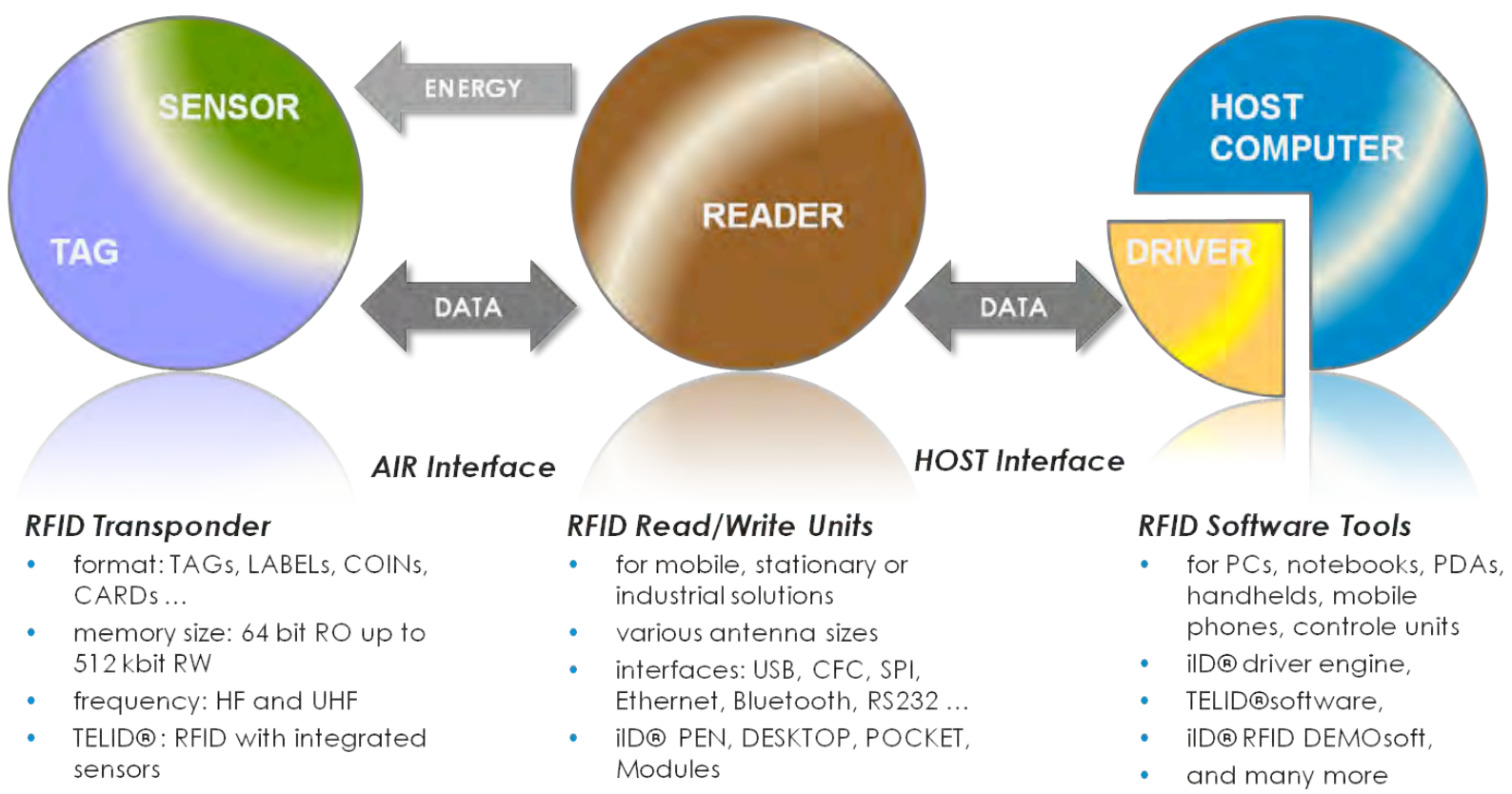

Abb. 1: Systemstruktur einer RFID-Lösung, der RFID-Transponder wird zum Sensor-TAG

Variiert werden dann nur noch möglicherweise Hardwaretreiber und natürlich die Applikationssoftware entsprechend der eingesetzten Sensorik sowie der geforderten Messaufgabe.

In Tab. 1 wird eine prinzipielle Übersicht gegeben, die eine Klassifizierung drahtloser Sensorik erlaubt. An erster Stelle steht die physikalische Größe, die gemessen werden soll. Das nächste wichtige Kriterium stellt die Realisierungsart des Wireless Interfaces sowie der Energieversorgung dar. Für die zu beschreibende Lösung eines passiven Schwingungssensorsystems kommt ausschließlich das RFIDInterface und der direkte Energie Transfer mittels Radiofrequenzen in Frage.

In Abhängigkeit von der Anwendung des Sensors sowie der gestellten Messaufgabe sind diverse Funktionalitäten umsetzbar. Basiert die Lösung auf der RFID-Technologie sind solche Funktionalitäten 
wie Identifikation, Datensicherheit und Ortsbestimmung mittels RTLS leicht umsetzbar oder meist automatisch in der Systemlösung vorhanden.

\begin{tabular}{|c|c|c|c|c|c|}
\hline Sensoren & \multicolumn{2}{|c|}{ Wirless Interface } & \multicolumn{2}{|c|}{$\begin{array}{l}\text { Energje- } \\
\text { Quelle/Speicher }\end{array}$} & Funktionalität \\
\hline Druck & \multirow[t]{3}{*}{ RFID } & LF & \multirow{2}{*}{$\begin{array}{l}\text { Energie } \\
\text { Transfer }\end{array}$} & RFID & Identification \\
\hline Temperatur & & $\mathrm{HF}, \mathrm{NFC}$ & & Laser & Data Security \\
\hline Feuchte & & UHF & \multirow{4}{*}{$\begin{array}{l}\text { Energy } \\
\text { Harvesting }\end{array}$} & Schwingung & Measure Only \\
\hline Leitfähigkeit & \multirow[t]{4}{*}{ RFDC } & Zigbee & & Solar & Limit Detection \\
\hline Kraft & & Bluetooth & & Wärme & Event Logger \\
\hline Schwingung & & WLAN & & Schall & Data Logger \\
\hline Neigung & & WWAN & \multirow{3}{*}{$\begin{array}{l}\text { Energie } \\
\text { Speicher }\end{array}$} & Kondensator & Sensor Analyzer \\
\hline \multirow[t]{3}{*}{ Füllstand } & SAW & $\mu$-wave & & Akkumulator & Mesh Network \\
\hline & US & ultrasonic & & Batterie & RTC \\
\hline & IR & IrDA & & & GPS \\
\hline
\end{tabular}

Tab. 1: Klassifizierungsmöglichkeit drahtloser Sensoren und Sensorsystemen

Bei Anwendung der passiven Transpondertechnik sind solche Funktionalitäten wie Logging, Networking, Limit Detection und der Betrieb einer Real Time Clock ausgeschlossen. Aus diesem Grund stellt die Schwingungsmessung eine besondere Herausforderung mittels passiver Technik dar, da es nicht genügt einen einzigen Meßwert zu erfassen.Die Schwierigkeit besteht hierbei darin, eine Messkurve energiesparend mit möglichst hoher Taktrate aufzuzeichnen und in kürzester Zeit zu übertragen.

Transponder bestehen grundsätzlich aus den Komponenten Antenne, RFID Frontend, Spannungsstabilisierung, Ablaufsteuerung und Datenspeicher meist in Form eines nichtflüchtigen Speichers.

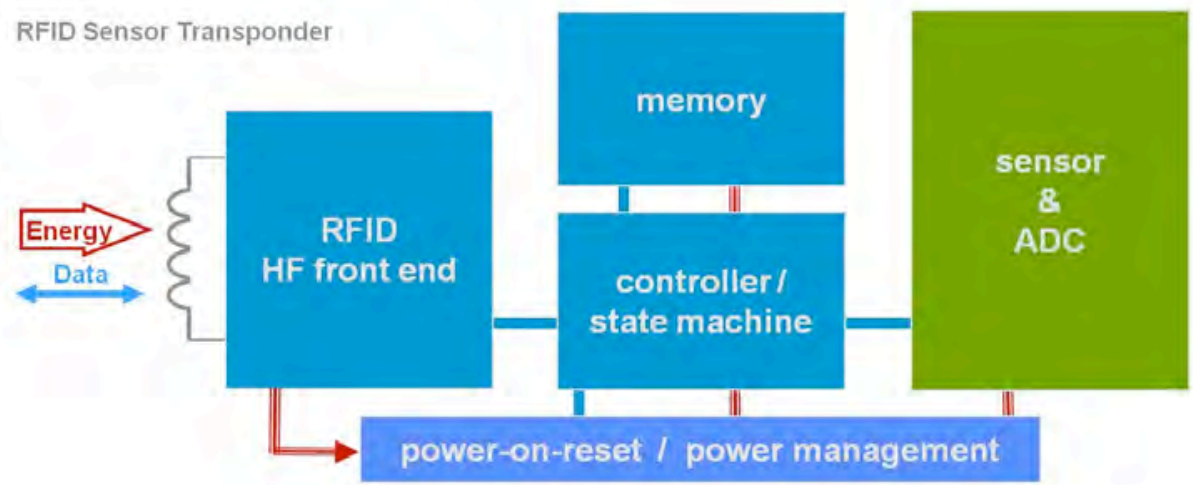

Abb. 2: Blockschema eines passiven Sensor-Transponders

Für den Fall eines passiven Sensor-Transponders wird meist eine umfangreichere Ablaufsteuerung, die im speziellen Fall durch einen freiprogrammierbaren Mikro-Controller vorgenommen wurde und ein aufwendigeres Power Management inklusive eines Energiezwischenspeichers in Größe einiger $\mu F$ nötig.

Seitens der RFID-Technologie stehen verschiedene Systeme zur Verfügung. Aus Leistungsverbrauchsgründen und optimalen Datenraten erweist sich die HF-Technik basierend auf dem Standard ISO 14443 als zielführend. Mit ihr sind in einem Kommunikationsbereich von einigen Zentimetern bis zu $10 \mathrm{~mW}$ Leistungsübertragung und Datenraten bis $424 \mathrm{kbps}$ möglich. Betrachtet man allerdings das für eineordentliche Schwingungsmessung im Industriebereich notwendige Frequenzspektrum von 0 bis $500 \mathrm{~Hz}$, wird sehr schnell deutlich, dass auch bei Reduzierung der Anforderungen und einer Digitalisierungsauflösung von nur 8 bit eine online-Übertragung der Daten nicht möglich ist. Es ist vielmehr notwendig die Schwingungsmessdaten im Transponder zwischenzuspeichern. 
Betrachtet man den normalerweise verfügbaren EEPROM Datenspeicher eines Transponders, so muss man feststellen, dass die zur Verfügung stehenden minimalsten Schreibzeiten von einigen $\mu$ s und die Endurance von kleiner 10.000 Zyklen nicht ausreichen. Der Datenspeicher wurde deshalb um einen RAM Baustein mit einer Speicherkapazität von 6 kbit erweitert. In ihm werden die digitalisierten Messwerte eines Schwingungspaketes mit 8 bit Genauigkeit zeitsynchron gespeichert, bevor sie über das AIRInterface zum Reader übertragen werden. Das heißt, Power on, Messung des Schwingungspaketes und Datenübertragung über das AIR-Interface sind zeitsequentielle Prozesse.

Entscheidend für die sensorische Umsetzung war die Verfügbarkeit eines miniaturisierten und extrem stromarmen Sensorchips. Durch die Firma Bosch Sensortec werden verschiedene 3D Beschleunigungssensoren mit digitaler Signalverarbeitung angeboten, geeignet erschien der Typ BMA150 [8].

Der typische Stromverbrauch dieses Chips liegt typisch bei $200 \mu \mathrm{A}$ bei 2,5 V Betriebsspannung.Ferner weist der Sensor folgende Daten auf:

$\begin{array}{ll}\text { Typ: } & \text { Bosch BMA150 (3D-Beschleunigun } \\ \text { Lage des Sensors: } & \text { asymmetrisch auf der Leiterplatte } \\ \text { Versorgungspannung: } & 2,4 \mathrm{~V} \text { bis } 3,6 \mathrm{~V} \\ \text { Package: } & 12 \mathrm{Pin} \mathrm{LGA} \\ \text { Messbereich: } & \pm 2 \mathrm{~g} / \pm 4 \mathrm{~g} / \pm 8 \mathrm{~g} \text { einstellbar } \\ \text { Bandbreite: } & 25 \mathrm{~Hz} \ldots 1500 \mathrm{~Hz} \text { einstellbar } \\ \text { Abtastrate: } & \max .1,95 \mathrm{kHz} \\ \text { Interface: } & \mathrm{I} \mathrm{C} / \mathrm{SPI} \\ \text { Stromverbrauch: } & \mathrm{Standby:} 2 \mu \mathrm{A} \\ \text { Normal Mode: } & \max .290 \mu \mathrm{A} \\ \text { Auflösung: } & 8 \mathrm{bit} \mathrm{bzw} .10 \mathrm{bit} \\ \text { Offset: } & \max .+150 \mathrm{mg} \\ \text { Output noise theoretisch: } & 0,5 \mathrm{mg} / \sqrt{\mathrm{Hz}} \\ \text { Temperaturbereich: } & -40^{\circ} \mathrm{C} \ldots+85^{\circ} \mathrm{C}\end{array}$

Die Größen Messbereich, Abtastrate, Bandbreite und Messintervallänge sind über das AIR-Interface programmierbar. Die gesamte Funktionalität des drahtlosen 3D Beschleunigungsmesssystems ist in einem Gehäuse mit einem Durchmesser von $15 \mathrm{~mm}$ und einer maximalen Dicke von $3 \mathrm{~mm}$ untergebracht. Die Abbildungen 3 und 4 zeigen das Produkt von Ober- und Unterseite sowie im offenen Zustand.

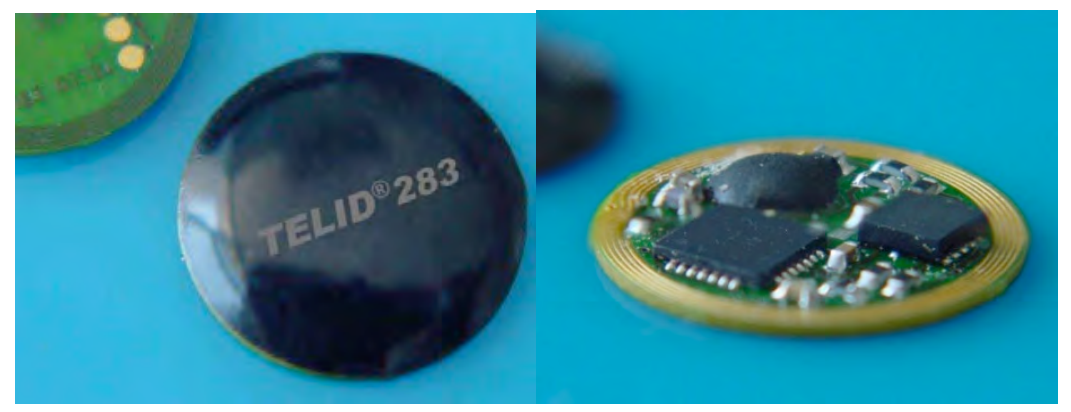

Abb. 3: passiver Schwingungstransponder TELID283

Abb. 4: TELID283 ohne Gehäuse

Erste Messungen und Erprobungen haben realisierbare Schwingungsmessungen im Frequenzbereich von 0 bis $500 \mathrm{~Hz}$ bei ca. 0,01 g Auflösung ergeben. Die gesamte Meßzeit dauert je nach eingestellter Parametrisierung zwischen 3 und 10 s. Die Kommunikationsabstände bei Verwendung eines standardmäßigen RFID Readers iID M30 HEAD liegen bei ungefähr $2-3 \mathrm{~cm}$. Die Masse des TELID283 liegt bei 0,5 g. Zur Zeit werden Untersuchungen vorgenommen, die eine Montage des TELID283 auch auf Metallobjekten erlauben. Die Probleme bei der Anwendung von TAGs auf Metall sind bekannt und lösbar.

Zur praktikablen Ausführung von Schwingungspaketmessungen wurde eine spezielle Anwendersoftware für Windows entwickelt. Sie gestattet die Parametrisierung des Systems und erlaubt eine komfortable Ausführung von Schwingungsmessungen. Die Daten werden zunächst in einem Datenfile hinterlegt und graphisch angezeigt. Zur weiteren Verarbeitung können diese in andere Analyseprogramme exportiert werden. 
Abb. 5 zeigt ein Schwingungspaket gemessen auf einer Lautsprechermembran mit einer Sinusansteuerung von ca. $50 \mathrm{~Hz}$. Die Membran orientiert dabei parallel zur Erdoberfläche.

Es sind eindeutig auch Lateralschwingungen der Membran feststellbar.

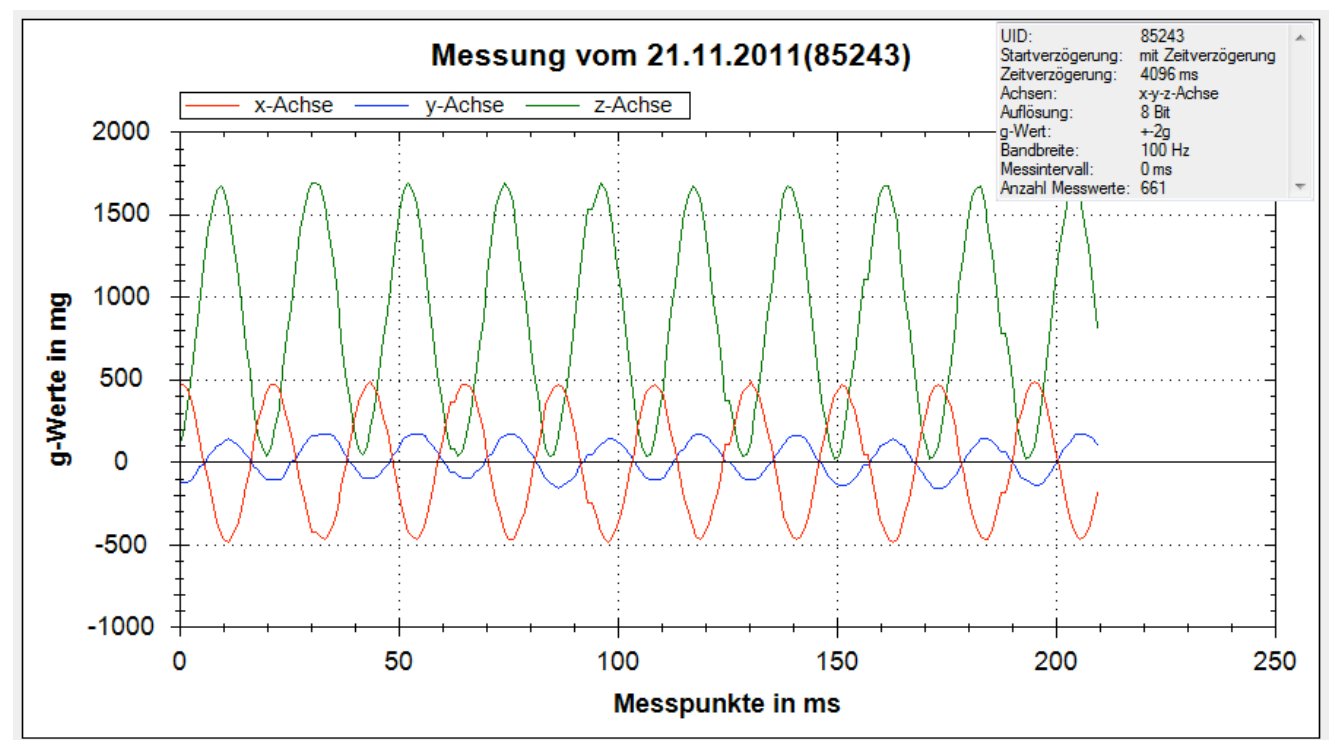

Abb. 5: 3D Schwingungsmessung an einer Lautsprechermembran mittels TELID283 als Sensor Transponder und iID M30 HEAD als RFID Reader

Prinzipiell kommunizieren die winzigen Sensorsysteme mit Standard HF Readern und üblicher mobiler Datentechnik wie Industrie Hand Helds, Smart Phones oder Notebooks.

Die Vorteile der passiven Sensortransponder gegenüber konventioneller kabelgebundener Technik oder aktiven, batteriegestützten Loggern und Funksendern liegen insbesondere in der geringen Masse der eigentlichen Meßvorrichtung, einer kleinen Baugröße. Ferner liefern sie, da sie am Meßobjekt verbleiben gleichzeitig eine eindeutige Identifikation der Meßstelle und ein ausgezeichnete Reproduzierbarkeit, da der Anspekt der Meßankopplung und die Einwirkung von Leitungen während der Messung entfallen.

Die RFID-Technik ist auch unter raue Umgebungsbedingungen einsetzbar. Standardschnittstellen erlauben eine preisgünstige Auswertetechnik.

Praktische Einsatzfelder solcher Sensoren wären im Industriebereich zur Maschinenkontrolle oder bei der Wartung und Instandhaltung zu sehen. Ein wesentlicher Vorteil solch einer drahtlosen Messung liegt in der störfreien Auskopplung der Schwingungsmessgröße.

\section{References}

[1] Jurisch, Reinhard: „Transponder mit integrierter Sensorik“, Elektronik, Fachzeitschrift für industrielle Anwender und Entwickler, 1. September 1998, Nr. 18, 76-79

[2] microsensys GmbH: „TECHNOLOGY NEWS INTERNATIONAL“, Journal of the Promotion of Technology, March 1998, Vol.1, 24

[3] Jurisch, R. : "Principles of Contactless RF-Transponders with Integrated Sensors"; Sensor 1999, Nürnberg, $9^{\text {th }}$ International Conference, Vol. 2, 221-226

[4] Finkenzeller, K.: RFID-Handbuch, Grundlagen und praktische Anwendungen induktiver Funkanlagen, 3. Auflage, München, Wien 2002

[5] P. A. Hagedorn: "Analyse und Design von RFID-Systemen und RFID-Sensorsystemen“, Studienarbeit, Juni 2006, Fachbereich Informatik, Universität Kaiserslautern 
[6] A. P. Sample, D. J. Yeager, A. V. Mamishev: „Design of an RFID-Based Battery-Free Programmable Sensing Platform", IEEE Trans. on Instr. and Measurem., Vol. 57, No. 11, Nov. 2008

[7] Raumedic: "Gehirndruckmessung”, Sept 2009, RFID im Blick, Vol. 3, 23-24

[8] Bosch Sensortec: „BMA150 digital, triaxial acceleration sensor”, Data sheet, 30. October 2008 